\section{P1-S2.55 HIGH INCIDENCE OF HIV AND LOW HIV PREVENTION COVERAGE AMONG MEN WHO HAVE SEX WITH MEN IN MANAGUA, NICARAGUA}

doi:10.1136/sextrans-2011-050108.112

\author{
${ }^{1} \mathrm{~F}$ Hernandez, ${ }^{1} \mathrm{~N}$ Arambú, ${ }^{1} \mathrm{~B}$ Alvarez, ${ }^{1} \mathrm{~L}$ Romero, ${ }^{1,2} \mathrm{~J}$ GoinsEBeteta, ${ }^{1,2,3} \mathrm{G}$ Paz-Bailey, \\ ${ }^{1} \mathrm{~S}$ Morales. ${ }^{1} \mathrm{Del}$ Valle University of Guatemala, Guatemala, Guatemala; ${ }^{2}$ Ministerio de \\ Salud de Nicaragua, Managua, Nicaragua; ${ }^{3}$ TEPHINET, Atlanta, Georgia, USA
}

Background High rates of HIV and sexually transmitted infections (STI) are found in men who have sex with men (MSM) in Nicaragua. This study investigated HIV, STI, and risk behaviours in MSM. Methods From 9/2009 to 2/2010, MSM 18 years or older who gave written informed consent and reported having anal sex with a man in the last year were recruited using respondent-driven sampling in Managua. The survey process included audio computer assisted survey instruments (ACASI) and face-to-face interviews followed by STI counselling and specimen collection for HIV (Uni-Gold Recombigen ${ }^{\circledR}$, Determine ${ }^{\circledR}$, HIV Elisa and Western Blot), HSV-2 (Elisa IgG Focus ${ }^{\circledR}$ Technology, Inc), and syphilis testing (RPR/ TPPA). Gonorrhoea, Chlamydia, Mycoplasma genitalium and Trichomonas were tested by PCR. The BED IgG capture enzyme immunoassay was applied to HIV positive specimens to detect recently-acquired HIV infections, used to estimate HIV incidence. Population proportions and $95 \%$ CIs were calculated and adjusted for RDS-sampling weights. Incidence estimates were adjusted using a locally-established false-recent rate for the assay. Data analyses were conducted in STATA 9.0 and RDSAT 6.1.

Results Respondents self-identified as bisexual (50\%), gay (41\%), heterosexual (1\%) or transgender (8\%). In the last year, 34.5\% had one or more stable partner and $66 \%$ had occasional partners. Sex with women during lifetime was reported by $66 \%$ and $40 \%$ in the last year. Consistent condom use (last 30 days) was reported at 31\% with stable male partner, $12 \%$, with stable female partner, $38 \%$ with occasional partner, and $38 \%$ with clients. Condom use in last sex was $62 \%$. Overall, $36 \%$ had received peer-driven outreach for HIV prevention and $64 \%$ had been tested for HIV in the last year. HIV prevalence in Managua was determined to be $7.5 \%$ (CI $4.5 \%$ to $11 \%$ ), incidence $2.9 \%$ (CI $0.2 \%$ to $5.6 \%$ ). Herpes simplex 2 was the most prevalent STI at $39.9 \%$ (CI $34.4 \%$ to $46 \%$ ) see Abstract P1-S2.55 Table 1.

Conclusions The 2003 Multicentric Study found a similar prevalence in Managua among MSM at 9.4\% (CI 5.3\% to 14.8\%). Condom use is low with all types of partners, but lowest with female partners. The population's high HIV incidence, coupled with low access to

Abstract P1-S2.55 Table 1 HIV and STI prevalence, men who have sex with men, ECVC Nicaragua, 2009

\begin{tabular}{|c|c|c|c|}
\hline & \multicolumn{3}{|c|}{ Managua } \\
\hline & $\mathbf{N}$ & n & $\%(95 \% \mathrm{Cl})$ \\
\hline HIV & 639 & 50 & $7.5(4.5$ to 11.1$)$ \\
\hline Herpes simplex tipo 2 & 632 & 293 & $39.9(34.4$ to 46.0$)$ \\
\hline Syphilis & 641 & 52 & 6.6 (3.9 to 9.3$)$ \\
\hline Active syphilis & 641 & 26 & $2.9(1.3$ to 4.7$)$ \\
\hline Neisseria gonorrhoeae & 512 & 15 & $2.6(0.8$ to 4.7$)$ \\
\hline N gonorrhoeae (urine) & 643 & 3 & $1.0(0$ to 2.1$)$ \\
\hline$N$ gonorrhoeae (rectal swab) & 511 & 12 & $1.4(0.4$ to 2.6$)$ \\
\hline Chlamydia trachomatis & 516 & 29 & $4.3(2.2$ to 6.2$)$ \\
\hline C trachomatis (urine) & 643 & 11 & $1.5(0.5$ to 2.9$)$ \\
\hline C trachomatis (rectal swab) & 511 & 18 & $2.3(1.0$ to 3.7$)$ \\
\hline Lymphogranuloma venereum & 511 & 4 & 0.7 (0 to 1.5$)$ \\
\hline Mycoplasma genitalium & 643 & 16 & $2.4(1.1$ to 3.9$)$ \\
\hline Trichomonas vaginalis & 643 & 4 & $0.7(0.1$ to 1.4$)$ \\
\hline HIV or any STI* & 530 & 147 & 24.9 (18.9 to 29.7$)$ \\
\hline Any STI & 526 & 110 & $17.6(12.5$ to 21.6$)$ \\
\hline
\end{tabular}

*Including HIV, Syphilis, Chlamydia, Gonorrhoea, Mycoplasma genitalium or Trichomonas. education, underlines the urgency of enhancing prevention activities among this population. Stronger strategies are needed to ensure that prevention, care, and treatment interventions reach this population.

\section{P1-S2.56 SEXUALLY TRANSMITTED DISEASES AMONG HIV POSITIVE MSM, PRIOR TO HCV INFECTION}

doi:10.1136/sextrans-2011-050108.113

L Apers, M Vandenbruaene, M Van Esbroeck, T Crucitti, E Florence. Institute of Tropical Medicine, Antwerp, Belgium

Background Hepatitis C (HCV) among HIV positive men who have sex with men (MSM) who deny injection drug use is likely to be acquired through sexual contact. Recurrent Sexually Transmitted Infections (STIs) are an indication of unsafe sexual practices and may be associated with HCV infection.

Methods In a retrospective study we analysed the laboratory data of 16 HCV positive/HIV positive MSM (cases), HCV diagnosis in 2010, and $32 \mathrm{HCV}$ negative/HIV positive MSM (controls), followed at the HIV/ STI clinic in Antwerp, Belgium. All laboratory confirmed STI episodes (syphilis, gonorrhoea, lymphogranuloma venereum, and rectal and urethral non-LGV chlamydial infections) were recorded since the date of presentation at our clinic, until the date of HCV diagnosis of the cases. Controls were selected from consecutive patients that consulted on the same day. Both cases and controls were regularly followed up at the clinic in view of their HIV infection. Syphilis screening is part of the three or four monthly laboratory check-up. The clinic is the first point of contact in case of signs or symptoms of an STI. STI episodes were defined on the basis of laboratory results-HCV infection was determined using a screening test and confirmed with a Line Immunoassay. Syphilis was diagnosed by RPR and TPPA seroconversion (primoinfection) or $\geq$ fourfold rise of RPR-titre (re-infection), gonorrhoea by culture and chlamydial infection by PCR. All STI episodes were treated according to CDC treatment guidelines 2010. We applied Poisson regression to assess the difference in STI-episodes per person-month of follow-up between cases and controls.

Results During 1049 and 1848 months of follow-up of the cases and controls respectively, 57 periods of STIs were diagnosed-32 periods of syphilis, 12 periods of gonorrhoea, 4 periods of lymphogranuloma venereum, 9 periods of rectal or urethral non-LGV Chlamydia trachomatis. The number of STI episodes per person-month followup was significantly higher for the cases as compared to the controls $(\mathrm{p}=0.005)$ see Abstract P1-S2.56 Table 1.

Conclusion The number of episodes of Sexually Transmitted Infections, prior to acquisition of HCV was significantly higher for HCV positive cases than for HCV negative controls. All patients were followed at the clinic for HIV positivity, as such an indication of risky sexual behaviour. The higher number of STIs in the history of the HCV cases may be associated with acquiring HCV. Cases and controls are enrolled in a larger study involving an in depth structured questionnaire that should shed light on behavioural factors that are associated with HCV infection. In Antwerp, a setting with a rising incidence of HCV among HIV positive MSM, an easy to recognise event such as recurrent STIs should lead to intensified screening for HCV and counselling of the patient.

Abstract P1-S2.56 Table 1

\begin{tabular}{llllllllll}
\hline & & $\begin{array}{l}\text { Months } \\
\text { of FU }\end{array}$ & $\begin{array}{l}\text { N } \\
\text { Syph }\end{array}$ & $\begin{array}{l}\text { N } \\
\text { Gono }\end{array}$ & $\begin{array}{l}\text { N LGV-C } \\
\text { trach }\end{array}$ & $\begin{array}{l}\text { N non- } \\
\text { LGV-C } \\
\text { trach }\end{array}$ & $\begin{array}{l}\text { Total STI } \\
\text { episo-des }\end{array}$ & $\begin{array}{l}\text { STI/ } \\
\text { 100MFU }\end{array}$ & p value \\
\hline Cases & 16 & 1049 & 15 & 10 & 2 & 4 & 31 & 2.9 & 0.005 \\
Controls & 32 & 1848 & 17 & 2 & 2 & 5 & 26 & 1.4 & \\
Total & 48 & 2897 & 32 & 12 & 4 & 9 & 57 & 2.0 & \\
\hline
\end{tabular}

